\title{
Space and Frequency Dependence of Nanocapacitor Array Sensors Response to Microparticles in Electrolyte
}

\author{
Andrea Cossettini ${ }^{\circledR}$, Member, IEEE, Cecilia Laborde, Denis Brandalise ${ }^{\circledR}$, Student Member, IEEE, \\ Frans Widdershoven, Serge G. Lemay ${ }^{\circledR}$, and Luca Selmi ${ }^{\circledR}$, Fellow, IEEE
}

\begin{abstract}
We present new experimental evidence and extensive numerical simulations of a few distinct fingerprints generated by dielectric and conductive microparticles in electrolyte environment on the capacitance spectra of nanoelectrode array sensors. Finite element simulations in good agreement with measurements allow us to identify unambiguously the physical origin of these features, and to illustrate their dependence on the system's geometrical and physical properties. In particular, we show that conductive particles induce a response with complex space and frequency dependencies, caused by the formation of an AC electrical double layer at the particle surface, and its interaction with the working and counter electrodes in the array. Furthermore, we highlight features that could lead to false-negative detection events in sensing applications. The theoretical predictions are confirmed by experiments on a state of the art CMOS pixelated nanocapacitor biosensor platform.

Index Terms - Nanocapacitor arrays, capacitance spectroscopy, electrical double layer.
\end{abstract}

\section{INTRODUCTION}

$\mathbf{H}$ IGH-FREQUENCY impedance spectroscopy (HFIS) is an attractive sensing principle to overcome the sensitivity limitations of conductance-based sensor devices [1], [2]. The Debye screening length, $\lambda_{D}$, is less than $1 \mathrm{~nm}$ at physiological salt concentration $(\approx 150 \mathrm{mM})$; thus, only small analytes in close proximity of the sensing surface, or small parts of

Manuscript received August 21, 2020; revised October 12, 2020; accepted October 14, 2020. Date of publication October 21, 2020; date of current version January 15, 2021. The work of Andrea Cossettini, Denis Brandalise, and Luca Selmi was supported by the Ministero dell'Istruzione, dell'Università e della Ricerca (MIUR) FLAG-ERA CONVERGENCE Project via the Consorzio Nazionale Interuniversitario per la Nanoelettronica (IUNET) consortium. The work of Cecilia Laborde and Serge G. Lemay was supported by the European Research Council (ERC) under Project 278801. The associate editor coordinating the review of this article and approving it for publication was Prof. Tarikul Islam. (Corresponding author: Andrea Cossettini.)

Andrea Cossettini was with the DPIA, University of Udine, 33100 Udine, Italy. He is now with the IIS, ETH Zürich, 8092 Zürich, Switzerland (e-mail: cossettini.andrea@spes.uniud.it).

Cecilia Laborde and Serge G. Lemay are with the MESA+ Institute for Nanotechnology, University of Twente, 7522 Enschede, The Netherlands.

Denis Brandalise and Luca Selmi are with the DIEF, University of Modena and Reggio Emilia, 41121 Modena, Italy.

Frans Widdershoven is with NXP Semiconductors, 5656 Eindhoven, The Netherlands, and also with the Bioelectronics Group, Department of Microelectronics, Delft University of Technology, 2628 Delft, The Netherlands.

Digital Object Identifier 10.1109/JSEN.2020.3032712 larger ones, can be detected in these conditions. Even worse, non-target molecules that are bound non-specifically to the electrode surface can give a much higher signal than target analytes that are captured a few nm above the surface. HFIS, instead, can extend the detection range to the scale of microns from the sensing surface [3]-[7]. In fact, above the dielectric relaxation frequency of the electrolyte, the $\mathrm{AC}$ electric field inside the Debye layer is almost the same as the electric field just above the Debye layer and there is thus no excess sensitivity for non-specifically bound non-target molecules.

Furthermore, HFIS lends itself to highly parallel implementation of sensing elements in standard CMOS technologies, which in turn enables the acquisition of large datasets, the implementation of accurate calibration and drift compensation algorithms, statistical analysis, pruning of bad electrodes, high spatial and temporal resolution, and even real-time imaging [8]-[11]. Integrated impedance spectroscopy platforms in CMOS have been presented in e.g., [9], [10], [12]-[22]. In particular, [9] demonstrated the detection and discrimination of dielectric and conductive microparticles at $50 \mathrm{MHz}$ and physiological salt concentrations.

Limited effort, however, has been devoted to quantitatively interpret the capacitance spectra and search for experimental signatures of different types of analytes. In [23], the possible existence of such signatures was put in evidence by modeling and simulation, suggesting that conductive microparticles 
may show an unexpected and counterintuitive dielectric-like behavior at low frequency. In [9], limited and preliminary experimental evidence was found that the capacitance response at high frequency changes sign when measured with HFIS platforms, but the physical origin of the phenomenon was not investigated further. The existence of frequencies of zero response however is quite relevant in practice because it may generate undesired false negatives in experiments at fixed frequency.

In this work we combine multidisciplinary competencies to extensively characterize and investigate the origin of these spectrally resolved findings. In particular, we consider the response of an HFIS array platform to conductive and dielectric micro-beads, which represent useful model systems for a variety of biologically interesting analytes (cells, bacteria, biomolecules, label particles, etc.).

\section{Materials And Methods}

The sensor platform, simulation model and experimental setups were described in [8]-[10]. Here we list only their main features for the sake of a self-contained paper.

\section{A. Biosensor Measurement System}

The platform implements, in $90 \mathrm{~nm}$ low-power CMOS technology, an array of $256 \times 256$ nanoelectrodes ( $180 \mathrm{~nm}$ diameter, $600 \mathrm{~nm} \times 890 \mathrm{~nm}$ pitch) including the selection, readout, A/D conversion, I/O, calibration and temperature monitoring circuits. Rows of working electrodes (WEs) are sequentially activated and the individual nanoelectrode capacitances are measured by high sensitivity charge-based capacitance measurement (CBCM) techniques [9], [24]. All electrodes in nonselected rows are $\mathrm{AC}$ grounded, to effectively constitute a large counter electrode (CE). The DC voltage between WEs and CEs is kept at $0 \mathrm{~V}$ to minimize electrodes' redox reactions.

Analytes captured on the electrodes perturb the local AC field, thus generating detectable steps in the capacitance vs. time traces [8]-[10]. The sensing events are expressed in terms of an effective capacitance variation [8], namely, $\Delta C=$ $C_{\mathrm{w} / \text { analyte }}-C_{\mathrm{w} / \mathrm{o} \text { analyte }}$ which accounts for both reactive and dissipative components. Pre- and post-measure calibration procedures enable accurate spectrally resolved measures in the 1-70 $\mathrm{MHz}$ range [9].

\section{B. Experiments}

Experiments were performed in $\mathrm{NaCl}$-enriched or diluted phosphate-buffered saline solutions (PBS) as appropriate to reach 10, 100 and $300 \mathrm{mM}$ ionic strength [8]. We inject dielectric and gold-coated polystyrene microparticles with radius $r_{p}=2.5 \mu \mathrm{m}$, let them sediment on the chip surface, and record the capacitance change over time while periodically cycling through different frequencies in the range 1.6-50 MHz.

\section{Numerical Models}

Three-dimensional control-volume finite element simulations of the array were carried out with ENBIOS [25]-[27].

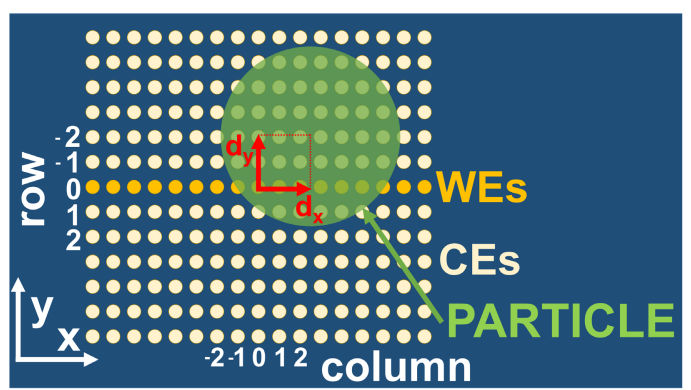

Fig. 1. Physical layout of the $13 \times 17$ nanoelectrodes simulation domain and definition of $d_{x}, d_{y}$. In simulations, unless otherwise stated, the $A C$ voltage is applied to an entire row (e.g., row 0), and the $A C$ currents of all nanoelectrodes in the row (WEs) are divided by the AC voltage, to determine the nanoelectrodes' AC admittances $Y_{A}$. All other nanoelectrodes are AC-grounded and act as counter electrodes (CEs).

ENBIOS solves the Poisson- Boltzmann (PB) and PoissonNernst-Plank (PNP) equations for the electrolyte in DC and small signal AC regimes, respectively, combined with model equations for the salinity, frequency and temperature dependence of the electrolyte permittivity, $\varepsilon_{e l}$ [28]. The compact sheet of counterions at the electrodes surface (so-called Stern or compact layer, $\mathrm{CL}$ ) is modeled as a thin dielectric film with thickness $t_{C L}=0.25 \mathrm{~nm}$ and permittivity $\varepsilon_{C L}=7 \varepsilon_{0}$ [29], [30]. We consider simulation domains of $13 \times 17,7 \times 7$ or $5 \times 5$ ideally-polarizable electrodes. As per the manufacturer specs, we assign a permittivity of $\varepsilon_{p}=2.6 \varepsilon_{0}$ and $\varepsilon_{p}=6.9 \varepsilon_{0}$ to dielectric and conductive beads, respectively; for the latter, we use a conductivity value of $\sigma_{p}=6.3 \cdot 10^{7} \mathrm{~S} / \mathrm{m}$. Simulations are reported in terms of the effective capacitance $C_{\text {eff }}=$ $\left|Y_{A}\right| / 2 \pi f$, where $Y_{A}$ is the single electrode admittance and $f$ is the frequency. $C_{\text {eff }}$ provides an adequate approximation to the actual switching capacitance measured by the CBCM on-chip detectors [8], [9].

\section{Results And Discussion}

Figure 1 shows a physical layout of the nanoelectrodes in the simulation domain. In the following, (row,column) $=(0,0)$ denotes the electrode located at the center of the simulation domain. The actual centroid of the spherical analyte is displaced by $\left(d_{x}, d_{y}, d_{z}+r_{p}\right)$ from the center of electrode $(0,0)$, where $r_{p}$ is the particle radius.

\section{A. Upper Frequency of Zero Response, $\Delta \mathrm{C}_{\text {eff }}=0$}

Figure 2 (top) shows the color map of the AC field modulus in the $y-z$ plane passing through the electrodes' center at low $(1 \mathrm{kHz})$ frequency. The corresponding $C_{\text {eff }}$ spectra at different salt concentrations are plotted as black curves with $\times$ symbols in Fig. 3. To first order, these graphs and curves can be interpreted with the aid of the well-known lumped element circuit of Fig. 2 (top) [3], [8], [9]. In the low frequency limit the field extends only inside the electrical double layer (EDL), and $C_{\text {eff }}$ tends to the surface capacitance $C_{S}$, given by the CL and EDL capacitance in series $\left(C_{C L}=\varepsilon_{C L} / t_{C L}, \quad C_{\mathrm{EDL}} \cong\right.$ $\left.\varepsilon_{e l} / \lambda_{D}\right)$. Consistently, the low frequency $C_{\text {eff }}$ increases for increasing salt concentration because $\lambda_{D}$ decreases.

$\begin{array}{ccccc}\text { Above a first cut-off frequency } f_{s} & = \\ /\left(2 \pi R_{E}\left(C_{E}+C_{\mathrm{EDL}}\right)\right) & \text { (where } C_{E} \text { and } R_{E} \text { are the }\end{array}$




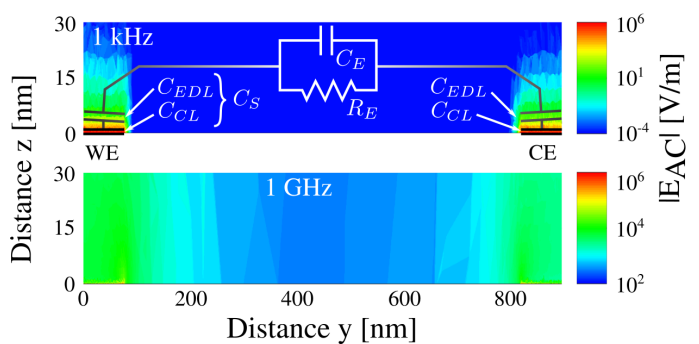

Fig. 2. Magnitude of the AC electric field between the center of one working electrode (left) and that of the adjacent counter electrode (right) on the yz plane. $300 \mathrm{mM} \mathrm{NaCl}$ electrolyte. Top: $f=1 \mathrm{kHz}$. Bottom: $f=1 \mathrm{GHz}$. The sketch on the top graph depicts a commonly used lumped element equivalent circuit model to interpret the electrode impedance response. $C_{S}$ represents the series of the compact layer, $C_{C L}$, and electrical double layer, $C_{\mathrm{EDL}}$, capacitances.

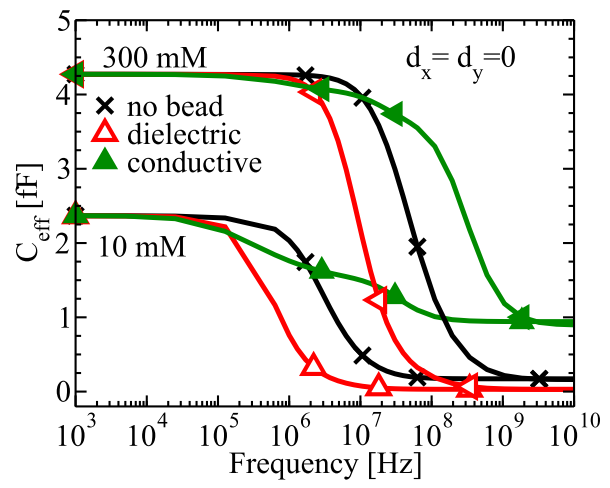

Fig. 3. $C_{\text {eff }}$ spectra at electrode $(0,0)$ without particles (black, $\left.x\right)$ and with a dielectric (red, $\Delta$ ) or conductive (green, $\mathbf{\Delta}$ ) bead, for $d_{x}=d_{y}=0$ and vertical elevation $d_{z}=10 \mathrm{~nm}, r_{p}=2.5 \mu \mathrm{m}$. Curves with dielectric beads are almost rigidly shifted to lower frequency. Instead, curves with and without conductive beads cross each other; this crossing entails opposite signs of $\Delta C_{\text {eff }}$ at low and high frequencies.

spreading capacitance and spreading resistance of the electrode in the electrolyte, respectively) the $\mathrm{AC}$ field starts to penetrate into the electrolyte, and the effective capacitance starts to decrease. Then, at frequencies exceeding the electrolyte dielectric relaxation cut-off frequency, $f_{c}=1 /\left(2 \pi R_{E} C_{E}\right)=\sigma_{e l} /\left(2 \pi \varepsilon_{e l}\right)$, the AC field inside the EDL drops to the bulk value just above the EDL, and the quasi-static Debye screening becomes ineffective (Fig. 2 and [8], [9], [23]). Eventually, the current mostly flows from the active electrode(s) to the nearest counter electrodes only. Therefore, in the high frequency limit, $C_{\text {eff }}$ tends to the geometry-dependent value set by the electrolyte spreading capacitance $C_{E}$ irrespective of the salt-concentration-dependent electrolyte spreading resistance $R_{E}$ (black lines of Fig. 3 converging to a unique value).

Figure 3 also shows the simulated $C_{\text {eff }}$ upon introduction of a dielectric or conductive particle right on top of the electrode $\left(d_{x}=d_{y}=0, d_{z}=10 \mathrm{~nm}\right)$. We observe that the low frequency capacitance limit remains the same regardless of the particle presence. A dielectric bead (red open symbols) lowers $f_{s}$ and $f_{c}$ by the same amount in relative terms (almost rigid leftward shift of the curve), thus generating a negative $\Delta C_{\text {eff }}$ over the whole spectrum [23].

Numerous experiments and simulations have been carried out with the nanocapacitor array platform to investigate this

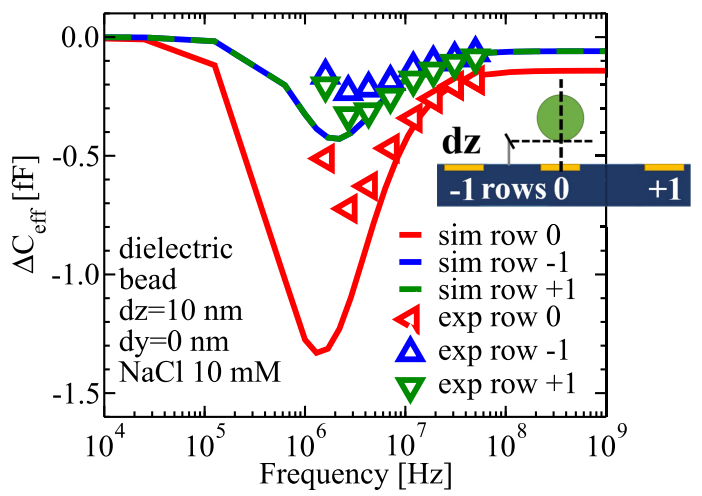

Fig. 4. Simulated (lines) and measured (symbols) $\Delta C_{\text {eff }}$ spectra at row 0 and at rows \pm 1 for a dielectric particle centered on the electrode $(0,0) . d_{z}=10 \mathrm{~nm} \gg \lambda_{D}, r_{p}=2.5 \mu \mathrm{m}$.

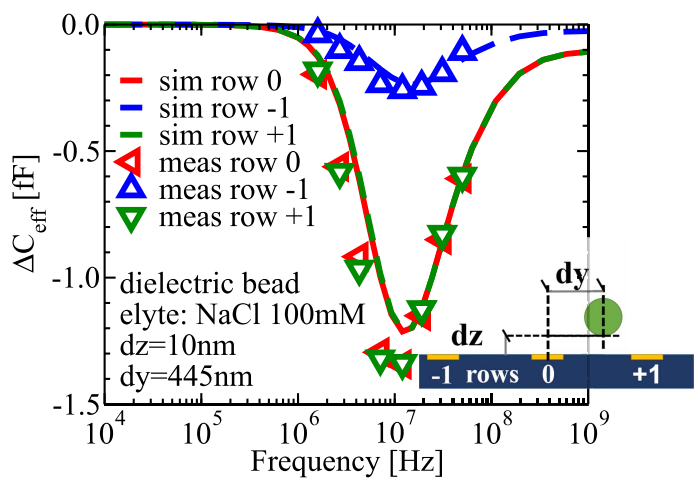

Fig. 5. Same as Fig. 4 for a dielectric bead in-between electrodes in the y-direction $\left(d_{y}=445 \mathrm{~nm}, d_{z}=10 \mathrm{~nm} \gg \lambda_{D}, r_{p}=2.5 \mu \mathrm{m}\right.$, $\mathrm{NaCl} 100 \mathrm{mM})$.

effect (by recording the particle response at different electrodes in the array) that allow us to explain in detail the findings.

Figure 4 shows new measurements of $\Delta C_{\text {eff }}$ as a function of frequency for three rows of electrodes. The \pm 1 rows adjacent to the central row feature the same trend as the one at electrode $(0,0)$, but with a smaller amplitude. Numerical simulations are in very good agreement with measurements, given the uncertainty in the actual particle elevation, $d_{z}$. This suggests that the negative effective capacitance change is mainly caused by the bead increasing the effective resistance between WEs and CEs. Indeed, when the AC excitation is on row " 0 ", the particle in $(0,0)$ effectively blocks current flow towards CEs in both row " +1 " and " -1 ", thus yielding a large negative response, whereas when $\mathrm{AC}$ excitation is on rows " \pm 1 " only the current toward row " 0 " is appreciably affected and the response is smaller in absolute value.

Measurements at rows +1 and -1 , however, are imbalanced, an effect easily explained by considering that in the experiments particles can land at some distance $d_{x}$ and $d_{y}$ from the electrode's center (Fig. 1). Consistently with this interpretation, measurements at an electrode where the imbalance between the first-neighbor rows is even larger, Fig. 5, are in good agreement with simulations where the particle is located in-between two electrodes $\left(d_{x}=0, d_{y}=445 \mathrm{~nm}\right)$.

Regarding conductive particles, Fig. 3 shows that for $f$ below a characteristic frequency $f_{z h}(\approx 2 \mathrm{MHz}$ at $10 \mathrm{mM}$ and $10 \mathrm{MHz}$ at $300 \mathrm{mM}$ ) we have $C_{\mathrm{w} / \text { analyte }}<C_{\mathrm{w} / \mathrm{o} \text { analyte }}$, 


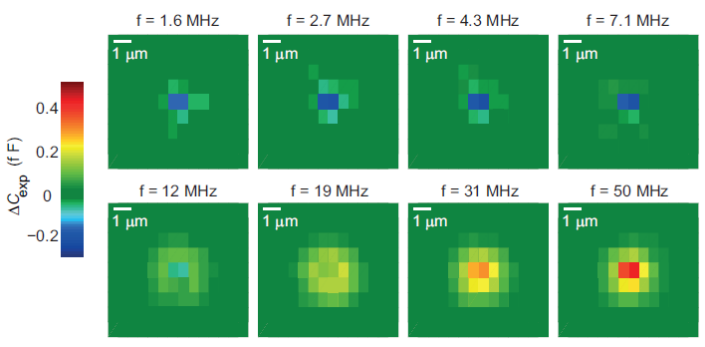

Fig. 6. Experimental $\Delta C_{\exp }$ for a conductive particle $\left(r_{p}=2.5 \mu \mathrm{m}\right)$ immersed in a $100 \mathrm{mM} \mathrm{NaCl}$ electrolyte at different frequencies. At low frequency, the capacitance change is negative, while at high frequency it becomes positive.

thus leading to $\Delta C_{\text {eff }}<0$, i.e. same sign as the dielectric bead case and opposite to the high frequency behavior. This effect, predicted by the compact model in [23] and to our knowledge first observed in [9], is here definitely confirmed by the new extensive experiments shown in Fig. 6 and Fig. 7, where $\Delta C_{\text {eff }}$ changes sign over frequency at several adjacent electrodes.

These results entail the existence of a frequency of zero response $f=f_{z h}$ such that $\Delta C_{\text {eff }}\left(f_{z h}\right)=0$. Such frequency of zero response and its presence at different electrodes is a remarkable feature of the nanoelectrode array spectral response to this analyte. Its existence bears two important consequences: firstly, at $f_{\mathrm{zh}}$ the sensor is blind to the particle presence and thus produces false negatives; secondly, only above $f_{z h}$ the transduction mechanism allows discrimination between dielectric and conductive analytes simply based on the sign of the $\Delta C_{\text {eff }}$ response.

Therefore, it is important to investigate the origin of this signature in quantitative detail, in order to assess the actual detection capabilities of HFIS sensors.

\section{B. Origin of the $\Delta \mathrm{C}_{\mathrm{eff}}=0$ Condition}

Firstly we observe that the crossing of the $C_{\text {eff }}$ traces with and without a conductive bead shown in Fig. 3, and hence the appearance of zeros of $\Delta C_{\text {eff }}$ in Figs. 6 and 7, corresponds to a sudden reduction (drop) of the effective capacitance spectrum with the bead followed by ranges where $C_{\text {eff }}$ is weakly dependent on frequency (hereafter denoted plateaus).

In order to understand the origin of this behavior, firstly we examined the role of the linear AC excitation pattern enforced at the nanoelectrodes, which breaks the symmetry of the otherwise nearly cylindrical/spherical system represented by the spherical beads on circular nanoelectrodes. However, the full row excitation is not the origin of the plateau and $\Delta C_{\text {eff }}\left(f_{z h}\right)=0$ condition, since simulations where only electrode $(0,0)$ acts as WE yield consistent results (not shown).

In fact, as shown in the following sections, depending on the salt concentration, particle size, position and charge, electrode radius and roughness (which in turn influence the formation of the CL and EDL) and number of active electrodes (WEs) in the central row, one or more of these plateaus appear, either distinct or gradually merged, but never entirely absent. The drops and plateaus can thus be regarded as additional

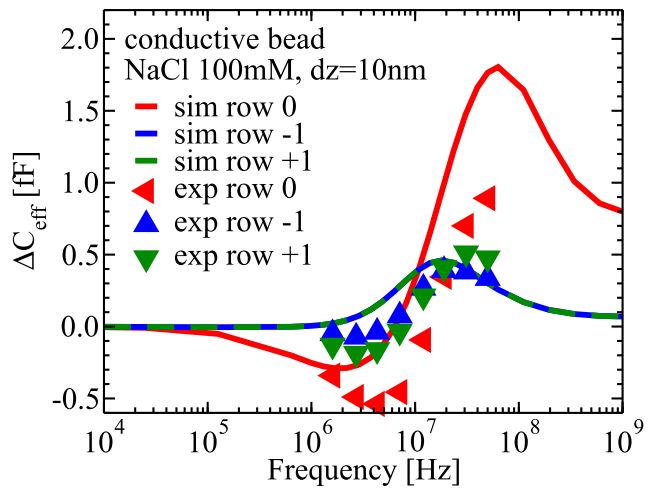

Fig. 7. Same as Fig. 4 for a for a conductive particle $\left(r_{p}=2.5 \mu \mathrm{m}\right.$, $100 \mathrm{mM} \mathrm{NaCl}$ electrolyte, $\left.d_{z}=10 \mathrm{~nm} \gg \lambda_{D}\right)$.

fingerprint signatures of the spectral response to the analyte, as discussed further below.

Careful examination of the simulations in Fig. 8 (a-e) shows how at low frequency all the voltage drop occurs in the nanoelectrode EDL so that, regardless of its conductive or dielectric nature, if the particle stands at $d_{z} \geq \lambda_{D}$, then it is essentially not reached by the excitation of the WE (red particle surface in Fig. 8a and 8b), i.e., its AC potential stays nearly equal to the counterelectrode potential of zero Volts. The electrolyte outside the EDL is effectively a short-circuit and thus the intensity of the AC current is essentially the same on all CEs, no matter of their distance to the WEs. Consequently, $C_{\text {eff }}$ at low-frequency is given by the series of $C_{C L}$ and $C_{\mathrm{EDL}}$ (namely, $C_{S}$ ) both with and without particles (i.e., $\left.\Delta C_{\text {eff }}\left(f \ll f_{s}\right)=0\right)$.

As frequency increases, the $\mathrm{AC}$ current starts to spread unevenly in the electrolyte. A dielectric bead obstructs the flow of current throughout most of its volume, increasing the spreading resistance and leaving the farther part of the bead's volume at much lower AC potential than the one facing the electrode (Fig. 8c). As regards the introduction of a conductive particle, at high frequency, the surface potential of the conductive particle appears well coupled to the WE potential (orange particle surface color in Fig. 8d), and even more so if the distance between particle and electrode is shortened (yellow color in Fig. 8e). This is because of a large EDL capacitance around the particle.

In fact, for a conductive particle, the metal short circuits part of the electrolyte resistance and capacitance, thus increasing $f_{s}$. However, at the same time a robust AC electrical double layer forms around the particle due to $\mathrm{AC}$ charge redistribution in the electrolyte and on the metal surface (Fig. 9, top left). This AC double layer corresponds to a high surface capacitance on the particle and in series to the current path toward the counterelectrode, that slightly reduces the $\mathrm{AC}$ current flow and the $C_{\text {eff }}$ and generates the first $C_{\text {eff }}$ drop with respect to the low frequency limit value. Incidentally, we note that a similar but much weaker (essentially negligible) AC EDL forms around the dielectric particle as well (Fig. 9, top right); its relevance for the capacitance response, however, is definitely smaller because of the insulating nature of the particle.

At even higher frequencies, the displacement current through the EDLs and the short circuit-like behavior of the 

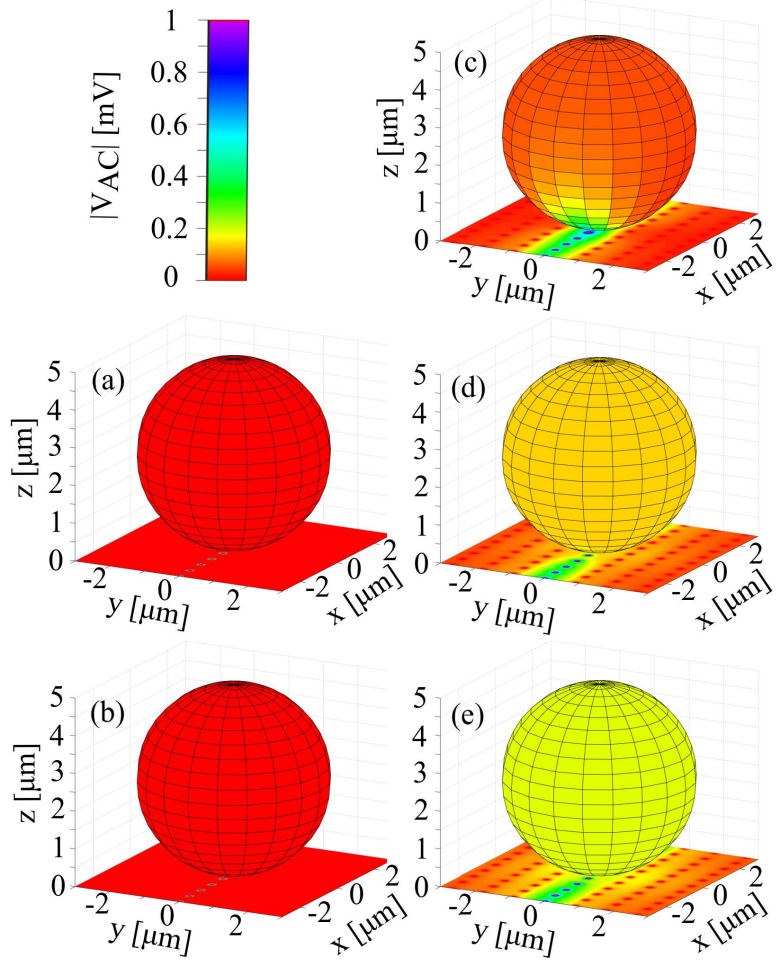

Fig. 8. Amplitude of the AC potential at the bead $\left(r_{p}=2.5 \mu \mathrm{m}\right)$ and at the array surfaces. (a) dielectric bead, $1 \mathrm{kHz}, d_{z}=10 \mathrm{~nm}$; (b) conductive bead, $1 \mathrm{kHz}, d_{z}=10 \mathrm{~nm}$; (c) dielectric bead, $50 \mathrm{MHz}, d_{z}=10 \mathrm{~nm}$; (d) conductive bead, $50 \mathrm{MHz}, d_{z}=10 \mathrm{~nm}$; (e) conductive bead, $50 \mathrm{MHz}$, $d_{z}=1 \mathrm{~nm}$. Electrolyte: $\mathrm{NaCl} 10 \mathrm{mM}\left(\lambda_{D} \approx 3 \mathrm{~nm}\right)$. At low frequency all the voltage drop occurs in the WE's EDL and the bead/array surface is at the same potential as the CEs (a-b). At high frequency the signal penetrates the EDL and reaches the beads (c-e). Conductive beads keep a constant voltage on their surface, enabling the signal to reach regions of the array far away from the electrode that were not reached before (d). This effect is further enhanced if the conductive bead is closer to the array (e). Here $d_{Z}<\lambda_{D} .13 \times 17$ nanoelectrodes array.

conductive particle, cause a decrease of $R_{E}$ and an increase of $C_{\text {eff }}$. The high frequency limit of $C_{\text {eff }}$ is thus higher, and it is reached at a higher frequency than without particles.

The role of the conductive particle EDL in the formation of the first capacitance drop is confirmed by additional simulations where we add a surface charge on the particle surface to alter its EDL (not shown). These results show unambiguously that the AC EDL on the particle surface is the root cause of the first $C_{\text {eff }}$ drop and corresponding zero in $\Delta C_{\text {eff. }}$.

\section{Effect of CEs Surface Conditions}

In the following, we examine the role of the CEs surface conditions by means of a set of simulations where the compact layer is selectively removed from some of the counterelectrodes, or the value of its dielectric permittivity/capacitance are changed. Note that by properly adjusting its thickness, extension and dielectric constant, the CL on the CEs can model a variety of diverse physical conditions: besides the Stern layer, it could also mimic, for instance, the presence of some extra undesired (disturbing) analytes or dirt or (possibly spatially non-uniform) functionalization layers on top of the electrodes. To continue our investigation, for convenience we momentarily

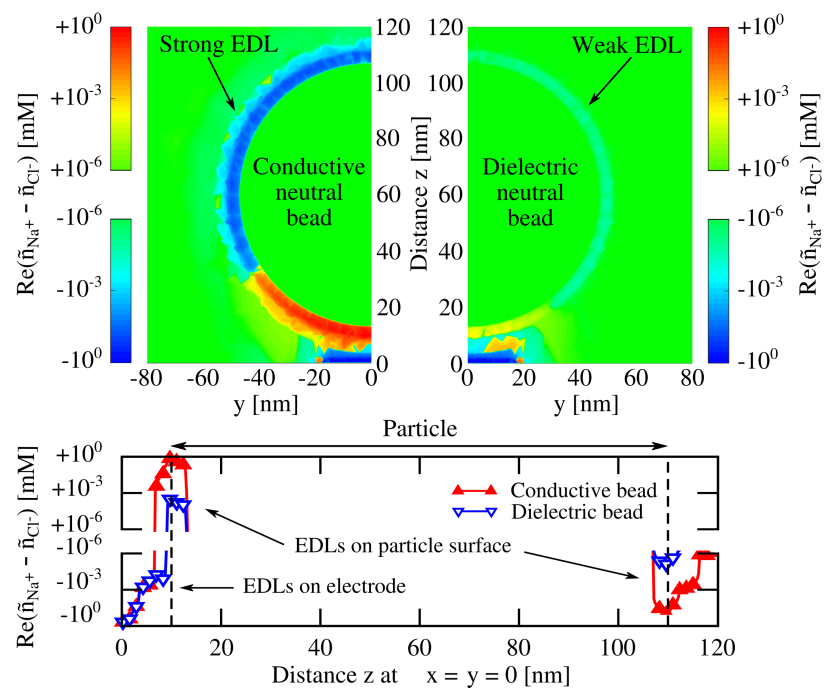

Fig. 9. Color maps of the real part of the net AC signed ion density $\operatorname{Re}\left(\tilde{n}_{\mathrm{Na}^{+}}-\tilde{n}_{\mathrm{Cr}}\right)$ (in signed log scales) for a conductive (top left) and dielectric (top right) particle in $300 \mathrm{mM} \mathrm{NaCl}$ electrolyte. The bottom graph reports the corresponding signed density profile along the $z$ direction at $d_{x}=d_{y}=0 \mathrm{~nm}$. Note the much weaker AC EDL of dielectric particles compared to conductive ones. The ion density decay length inside the particle is due to numerical interpolation. Scaled system with $r_{p}=50 \mathrm{~nm}, r_{e l}=15 \mathrm{~nm}, f=30 \mathrm{MHz}<f_{z h}$.

focus on smaller and more numerically manageable systems of $7 \times 7$ or $5 \times 5$ electrodes, considering particles with $r_{p}=1 \mu \mathrm{m}$, which still retain the essential physics of the more complex $13 \times 17$ array but saves substantial simulation time.

Figure 10 compares simulations of a $7 \times 7$ array with a conductive particle at $d_{z}=1 \mathrm{~nm}$ in $\mathrm{NaCl} 300 \mathrm{mM}$ (i.e., beyond the EDL of the electrodes), either including a dielectric compact layer (CL) on both WEs and CEs (sketch on the bottom right) or only on the row of working electrodes (sketch on the top right). In the range between the low-frequency and the high-frequency capacitance values, either one or two intermediate dips and plateaus appear, depending on the CEs' configuration.

The presence of a second drop and plateau in the frequency range between $f_{s}$ and $f_{c}$ and distinct from the first one discussed above are observed also in simulations where CLs of different dielectric permittivity are placed on peripheral rows at some distance from the WEs row (not shown).

These analyses illustrate how the properties of the CEs contribute to smoothen the $C_{\text {eff }}$ transitions between the low and high frequency limits. In fact, in a lumped element equivalent circuit view, each current density flow line from the WE toward different CEs has slightly different series capacitance and resistance, hence, different time-constants of the corresponding poles and zeros of the capacitance spectra. The CE area also plays a role in determining the amplitude and frequency of these drops. Simulations of a simple cylindrical domain with a single WE, a single $\mathrm{CE}$ and a conductive particle in-between, where only one current path exists between the WE and the CE, always yield a single intermediate dip and plateau (data not shown), confirming the point.

In summary, according to our analysis, we expect that in a quite general idealized scenario, the capacitance spectra 


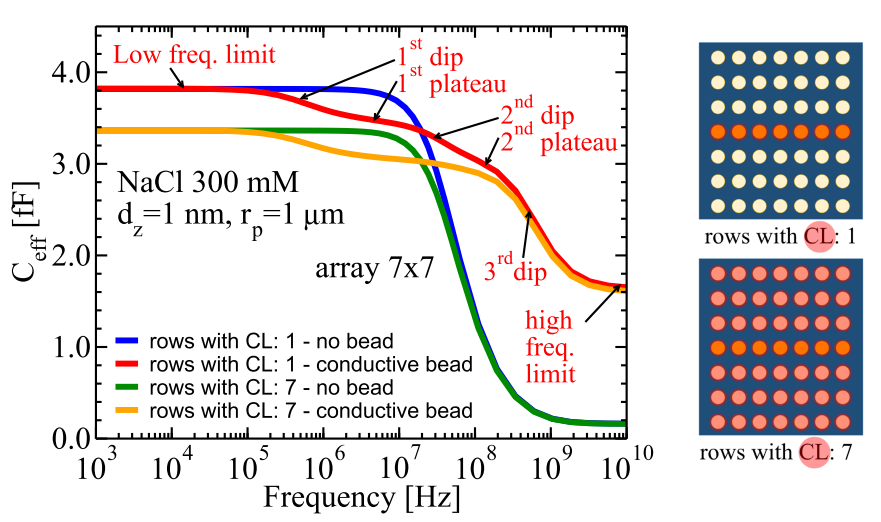

Fig. 10. $\quad C_{\text {eff }}$ spectra for conductive particles $\left(r_{p}=1 \mu \mathrm{m}\right)$ in $\mathrm{NaCl} 300 \mathrm{mM}$, located at $d_{z}=1 \mathrm{~nm} \geq \lambda_{D}$. An array of $7 \times 7$ electrodes is considered. A dielectric compact layer $(C L)$ is included either on all electrodes, or on only the electrodes of the active row. The lowfrequency capacitance limit is smaller in the first case because of the CL capacitance of the $(7-1) \times 7$ CEs which adds in series to the current lines originating from the WEs.

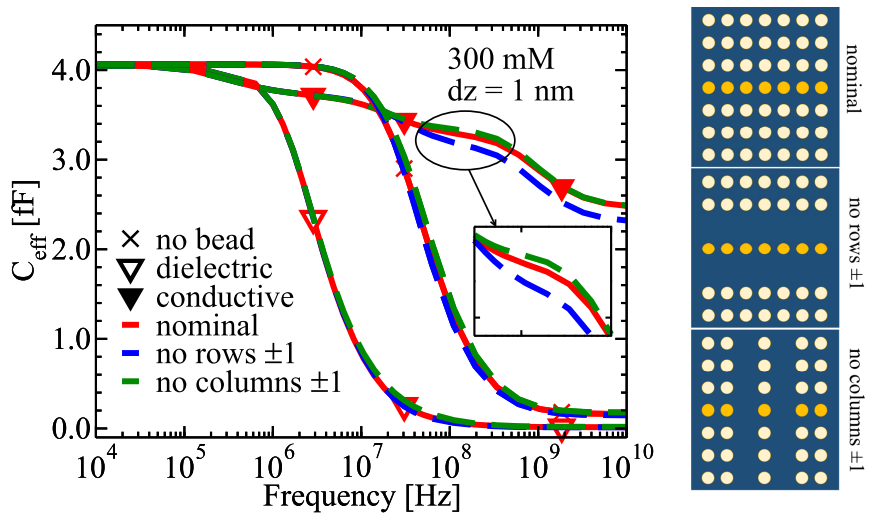

Fig. 11. $C_{\text {eff }}$ spectra for $r_{p}=1 \mu \mathrm{m}$ dielectric and conductive particles at $d_{z}=1 \mathrm{~nm} \geq \lambda_{D}$ removing some electrode rows or columns of a $7 \times 7$ array. Electrolyte: $\mathrm{NaCl} 300 \mathrm{mM}$. Row-wise activation. $\mathrm{CL}$ added on all rows except the outermost ones.

of conductive particles has three capacitance drops with two intermediate plateaus between the low-frequency and highfrequency limit of the response, possibly with a smoothing of the curves caused by the multiple time constants that variability and fluctuation sources cause in the system.

\section{Effects of Electrode Configuration and Current Paths}

Fig. 11 shows that by changing the spatial configuration of WEs and/or CEs the second drop and the asymptotic $C_{\text {eff }}$ value at high frequency are affected, while the spectra around the first drop remain unaltered. By removing rows of counterelectrodes next to the WE row, the current path between the WE and the CEs become longer, the series resistance increases and $C_{\text {eff }}$ decreases. On the contrary, by removing adjacent columns, the current of the WE spreads out, but the WE is still strongly coupled to its nearest neighbor CEs in the same column. Consequently $C_{\text {eff }}$ increases only slightly. These observations clearly indicate that the second drop is mostly affected by the geometry of the current distribution in the electrolyte which primarily reflects the working/counterelectrodes number, location and geometry. The first drop is quite insensitive to these factors, being due to the particle EDL

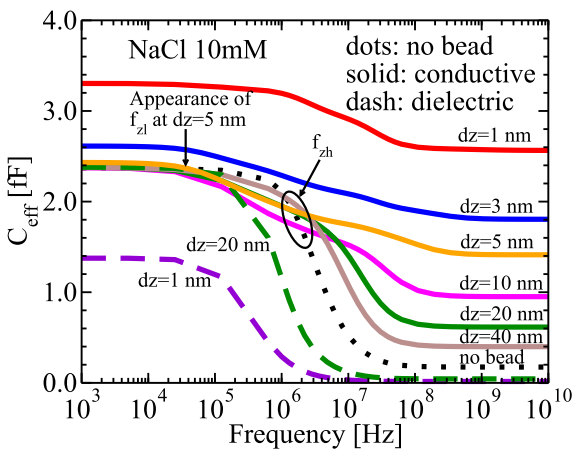

Fig. 12. $\quad C_{\text {eff }}$ spectra of a $r_{p}=2.5 \mu \mathrm{m}$ bead at different vertical displacements $\left(d_{x}=d_{y}=0\right)$ over a $13 \times 17$ array in $\mathrm{NaCl} 10 \mathrm{mM}$.

as shown above. This geometry-dependent sensing characteristic [31] is reasonable, since the frequency is large enough that the AC field considerably penetrates the electrolyte.

\section{E. Effect of Particle Elevation $\mathrm{d}_{\mathrm{z}}$}

Until now, results refer to $d_{z} \gg \lambda_{D}$. It is interesting to note that if the conductive particle comes closer to the electrode (decreasing $d_{z}$ ) while still not interfering with its EDL (e.g., $d_{z}$ from 40 to $10 \mathrm{~nm}$ in Fig. 12), then the first drop and the value of $f_{z h}$ become slightly larger because of the smaller electrolyte resistance and larger electrolyte capacitance in series with the EDL capacitance. In other words, if $d_{z}$ decreases, the $C_{\text {eff }}$ curve detaches from the low-frequency limit capacitance value at a smaller frequency, eventually intercepting the capacitance curve in the absence of particle (black line) at a higher $f_{z h}$.

As the particle's and WE's EDLs start to interact significantly (e.g., $d_{z}$ from 5 to $1 \mathrm{~nm}$ in Fig. 12) the low frequency limit of the capacitance curve is affected. Then, for conductive particles, the potential of the particle couples tightly to the electrode, and the particle tends to behave as a protrusion of the electrode (compare Fig. 8e to Fig. 8d). The effective area of the electrode and consequently its capacitance increase above the value without particle not only at high but also at low frequency. As a consequence, $f_{z h}$ (which was previously increasing for decreasing $d_{z}$ ) starts to decrease and, furthermore, an additional sign change of $\Delta C_{\text {eff }}$ appears at low frequency $\left(f_{z l}\right)$. For dielectric particles, instead, the interaction of the particle with the WE's EDL obstructs increasing portions of the current flowlines so that the low frequency capacitance is reduced and a larger $\left|\Delta C_{\text {eff }}\right|$ appears at low frequency.

Fig. 13 illustrates how $f_{z h}$ changes with the distance between the electrode and the analyte and the appearance of $f_{z l}$. Note that $f_{z l}$ may fall outside the measurable range or be hidden by numerical noise in simulations as well as in experiments. The two distinct frequencies of zero response tend to converge onto each other going toward smaller $d_{z}$, reaching the limit case of $f_{z h}=f_{z l}$ when the capacitance spectrum in the presence of the conductive particle is perfectly tangent to the capacitance spectrum in the absence of the particle. For even lower $d_{z}$, the two spectra do not intersect anymore (red and blue curves in Fig. 12), $C_{\text {eff }}$ for the conductive particle is always larger than $C_{\text {eff }}$ without analyte, and the frequencies 


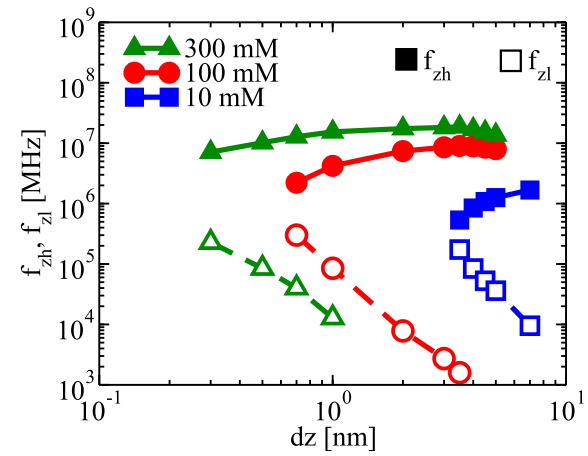

Fig. 13. Simulated frequencies of zero response of a conductive particle as a function of different vertical displacements $d_{z}$.

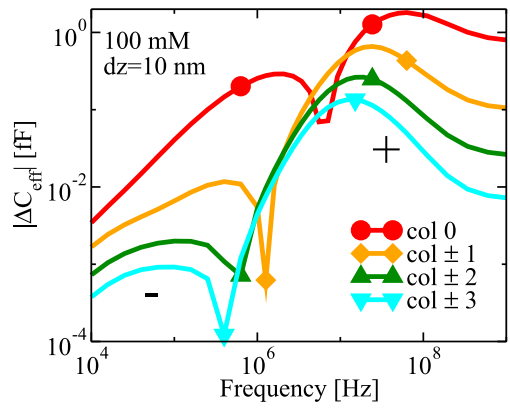

Fig. 14. Simulated $\Delta C_{\text {eff }}$ spectra as seen from different electrodes of the active row. $r_{p}=2.5 \mu \mathrm{m}$. The dips mark the values of $f_{z h}$ and the sign change of $\Delta C_{\text {eff }}$.

of zero $\Delta C_{\text {eff }}$ disappear. These results suggest the possibility to estimate the elevation of the particle $\left(d_{z}\right)$ by checking the separation between $f_{z h}$ and $f_{z l}$.

\section{F. Multi-Electrode Capacitance Profiles and Image}

Fig. 14 shows the simulated $\left|\Delta C_{\text {eff }}\right|$ spectra of a $r_{p}=$ $2.5 \mu \mathrm{m}$ conductive particle centered on electrode $(0,0)$ as detected at different electrodes on the same row. As a result of the complex, interdependent effects discussed above and consistently with Fig. 13, we see that $f_{z h}$ decreases for increasing column number. For a spherical particle, this entails a larger distance between the detection electrode and the particle, and consequently a larger resistance in series to the EDL capacitance. As a result, a frequency range exists where the particle response is negative, zero or positive, depending on the electrode under observation.

The above results allow us to fully interpret the response of WEs at different distance from the analyte as a function of position and frequency, as elucidated below.

Figures 15 and 16 compare $\Delta C_{\text {eff }}$ measurements and simulations of dielectric and conductive particles taken at different electrodes along the active row for a few frequency values. As expected, dielectric beads (Fig. 15) always yield a negative $\Delta C_{\text {eff }}$ and the peak response is centered, for each frequency, on the electrode which is nearest to the bead itself. Instead, conductive beads (Fig. 16) exhibit electrodes that have a larger response than the electrode $(0,0)$ nearest to the particle. This experimental behavior, qualitatively well reproduced by simulations, is a consequence of the shift of the $\Delta C_{\text {eff }}$ spectrum and of the corresponding $f_{z h}$ reported in Fig. 14 consistently with

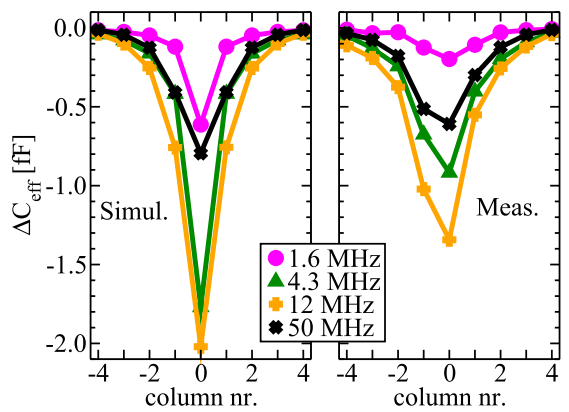

Fig. 15. Simulated (left, $d_{x}=d_{y}=0 \mathrm{~nm}, d_{z}=10 \mathrm{~nm}$ ) and measured (right) $\Delta C_{\text {eff }}$ for a dielectric particle as detected at the electrodes of the active row. $r_{p}=2.5 \mu \mathrm{m}, \mathrm{NaCl} 100 \mathrm{mM}, 13 \times 17$ array. $\Delta C_{\text {eff }}$ is in good agreement with simulations and, as expected, always negative.

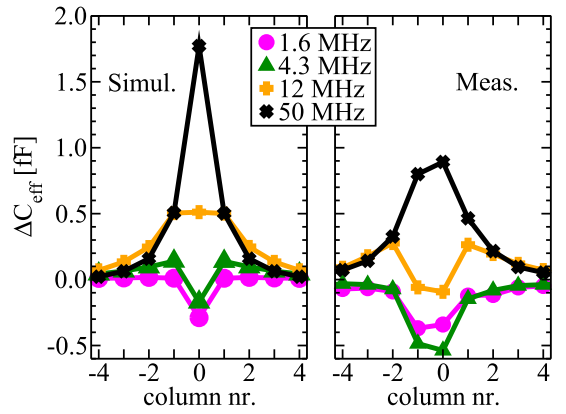

Fig. 16. Same as Fig. 15 for a conductive bead. $r_{p}=2.5 \mu \mathrm{m}$, $\mathrm{NaCl} 100 \mathrm{mM}, 13 \times 17$ array. The $\Delta C_{\text {eff }}$ sign change occurs at larger frequency for electrode 0 than for electrode 1 , consistently with the $f_{z h}$ in Fig. 14.

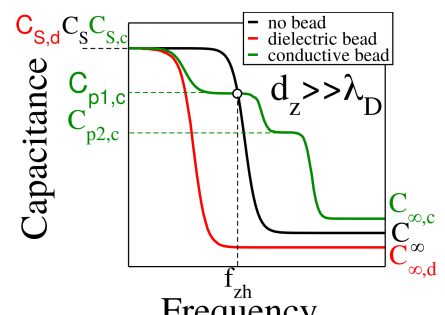

Frequency

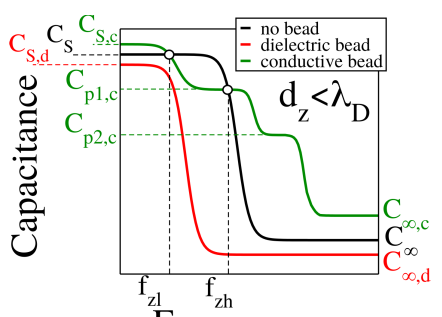

Frequency
Fig. 17. Summary sketches of $C_{\text {eff }}$ spectra without (black) and with a dielectric (red) or conductive bead (green), for $d_{z} \gg \lambda_{D}$ (left) or $d_{z}<$ $\lambda_{D}$ (right). The intermediate plateaus of the conductive bead's spectrum $\left(C_{p 1, c}\right.$ and $\left.C_{p 2, c}\right)$ result in a crossing with the spectrum without bead, which result in frequencies of zero $\Delta C_{\text {eff }}\left(f_{z l}\right.$ and $\left.f_{z h}\right)$.

the results of Fig. 13. In measurements the peaks are smoother than in simulations, likely because the particle is not exactly centered on the electrode.

\section{CONCLUSION}

We reported accurate measurements and extensive simulations of nanoelectrode capacitance that shed light on the spectral response of nanoelectrode arrays and thoroughly explain the observed frequencies of zero response to conductive particles. Fig. 17 summarizes our main findings in simplified sketches. In the high-frequency limit, the response to a conductive bead $\left(C_{\infty, c}\right)$ is always larger than the response in absence of a bead $\left(C_{\infty}\right)$; for the dielectric bead, instead, $C_{\infty, d}<C_{\infty}$. In the low frequency limit, and for a bead perturbing the electrode's EDL (right plot, $d_{z}<\lambda_{D}$ ), we have 
$C_{S, d}<C_{S}<C_{S, c}$. Differently, when the microparticle lies beyond the EDL boundary (left plot, $d_{z} \gg \lambda_{D}$ ), both $C_{S, d}$ and $C_{S, c}$ tend to $C_{S}$. In other words, the low-frequency limit of $C_{\text {eff }}$ is set mainly by the nanoelectrode CL and EDL capacitance; it is essentially the same with and without particles unless the bead lies within less than about one Debye length of the electrode. The high frequency limit $\Delta C_{\text {eff }}$ reflects the electrolyte volume substitution by the particle [23]: the low permittivity of a dielectric bead reduces the capacitance, whereas a conductive bead short-circuits a portion of electrolyte, thus increasing the capacitance. As a result, the high frequency limit of $\Delta C_{\text {eff }}$ has an opposite sign for dielectric and conductive neutral particles.

Furthermore, dielectric particles increase $R_{E}$ and shift $f_{s}$ and $f_{c}$ to lower frequency and thus generate a negative $\Delta C_{\text {eff }}$ at all frequencies. Instead, the EDL at the surface of a conductive particle generates a capacitance drop at intermediate frequencies, resulting in frequencies of vanishing $\Delta C_{\text {eff. For }} f$ values approximately in the $\left[f_{s}, f_{c}\right]$ range, $\Delta C_{\text {eff }}$ reflects the interaction of the WE with the nearby CEs through the analyte. As a result, the shape of the curve is sensitive to the CEs geometry and location, as well as to changes, variability or fluctuations at the CE surface. These effects can smear out the details of the single nanoelectrode response predicted by ideal calculations, especially for analyte dimensions comparable to a few times the nanoelectrode pitch.

Note that both the experimental and simulated results have been derived with reference to nearly ideal spherical beads. However, the physical origin and main dependencies of the newly identified spectral features are likely to remain the same also for particles of other shapes. Therefore, the results are useful to assist the interpretation of experimental capacitance data taken with nanoelectrode array sensors in more general conditions than those explored here. Furthermore, the analysis highlights the conditions where vanishingly small responses, i.e. false negatives, may appear in single frequency detection experiments. To avoid missing the possible presence of analytes on the nanoelectrodes, both multi-frequency measurements and neighbor nanoelectrodes should be considered. The conditions of the counterelectrodes should also be accounted for in the analysis of each nanoelectrode signal.

The results also suggest ways to exploit these distinct experimental signatures for physical/geometrical parameter extraction and metrology of small analytes (e.g., determination of $d_{z}$ ). Finally, they demonstrate the ability of the PoissonBoltzmann/Poisson-Nernst-Planck modeling framework to provide quantitative predictions of the nanoelectrode array response under various conditions. Besides giving new insight into nanoelectrode array capacitance measurements, these calculations can be used to generate databases of simulated capacitance images suited to assist the training of machine learning/deep learning algorithms for analyte identification, and to support the development of compact circuit models of the transduction mechanism of nanoelectrode array sensors.

\section{References}

[1] P. R. Nair and M. A. Alam, "Screening-limited response of nanobiosensors," Nano Lett., vol. 8, no. 5, pp. 1281-1285, May 2008, doi: 10.1021/ n1072593i.
[2] V. Kesler, B. Murmann, and H. T. Soh, "Going beyond the debye length: Overcoming charge screening limitations in next-generation bioelectronic sensors," 2020, arXiv:2007.13201. [Online]. Available: http://arxiv.org/abs/2007.13201

[3] A. J. Bard and L. R. Faulkner, Electrochemical Methods: Fundamentals and Applications, 2nd ed. Hoboken, NJ, USA: Wiley, 2001.

[4] A. Matsumoto and Y. Miyahara, "Current and emerging challenges of field effect transistor based bio-sensing," Nanoscale, vol. 5, no. 22, pp. 10702-10718, 2013, doi: 10.1039/c3nr02703a.

[5] G. S. Kulkarni and Z. Zhong, "Detection beyond the debye screening length in a high-frequency nanoelectronic biosensor," Nano Lett., vol. 12, no. 2, pp. 719-723, Feb. 2012, doi: 10.1021/n1203666a.

[6] F. Pittino, L. Selmi, and F. Widdershoven, "Numerical and analytical models to investigate the AC high-frequency response of nanoelectrode/SAM/electrolyte capacitive sensing elements," Solid-State Electron., vol. 88, pp. 82-88, Oct. 2013, doi: 10.1016/j.sse.2013.04.016.

[7] S. Ingebrandt, "Bioelectronics: Sensing beyond the limit," Nature Nanotechnol., vol. 10, no. 9, pp. 734-735, 2015, doi: 10.1038/nnano.2015. 199.

[8] C. Laborde et al., "Real-time imaging of microparticles and living cells with CMOS nanocapacitor arrays," Nature Nanotechnol., vol. 10, no. 9 , pp. 791-795, Sep. 2015, doi: 10.1038/nnano.2015.163.

[9] F. Widdershoven et al., "A CMOS pixelated nanocapacitor biosensor platform for high-frequency impedance spectroscopy and imaging," IEEE Trans. Biomed. Circuits Syst., vol. 12, no. 6, pp. 1369-1382, Dec. 2018, doi: 10.1109/TBCAS.2018.2861558.

[10] F. Widdershoven et al., "CMOS biosensor platform," in IEDM Tech Dig., Dec. 2010, pp. 816-819, doi: 10.1109/IEDM.2010.5703484.

[11] B. Stadlbauer et al., "Bayesian estimation of physical and geometrical parameters for nanocapacitor array biosensors," J. Comput. Phys., vol. 397, Nov. 2019, Art. no. 108874, doi: 10.1016/j.jcp.2019.108874.

[12] A. Manickam, A. Chevalier, M. McDermott, A. D. Ellington, and A. Hassibi, "A CMOS electrochemical impedance spectroscopy (EIS) biosensor array," IEEE Trans. Biomed. Circuits Syst., vol. 4, no. 6, pp. 379-390, Dec. 2010, doi: 10.1109/TBCAS.2010.2081669.

[13] C. C. Wong et al., "CMOS based high density micro array platform for electrochemical detection and enumeration of cells," in IEDM Tech. Dig., Dec. 2013, pp. 14.2.1-14.2.4, doi: 10.1109/IEDM.2013.6724628.

[14] V. Vijay et al., "High-density CMOS microelectrode array system for impedance spectroscopy and imaging of biological cells," in Proc. IEEE Sensors, Oct. 2016, pp. 1-3, doi: 10.1109/ICSENS.2016.7808761.

[15] C. M. Lopez et al., "A 16384-electrode 1024-channel multimodal CMOS MEA for high-throughput intracellular action potential measurements and impedance spectroscopy in drug-screening applications," in IEEE ISSCC Dig. Tech. Papers, Feb. 2018, pp. 464-466, doi: 10.1109/ISSCC. 2018.8310385.

[16] J. S. Park et al., "1024-pixel CMOS multimodality joint cellular sensor/stimulator array for real-time holistic cellular characterization and cell-based drug screening," IEEE Trans. Biomed. Circuits Syst., vol. 12, no. 1, pp. 80-94, Feb. 2018, doi: 10.1109/TBCAS.2017.2759220.

[17] G. Qu et al., "A $0.28 \mathrm{~m} \Omega$-sensitivity $105 \mathrm{~dB}$-dynamic-range electrochemical impedance spectroscopy soc for electrochemical gas detection," in IEEE ISSCC Dig. Tech. Papers, Feb. 2018, pp. 286-288, doi: 10.1109/ISSCC.2018.8310296.

[18] A. Susloparova, D. Koppenhöfer, X. T. Vu, M. Weil, and S. Ingebrandt, "Impedance spectroscopy with field-effect transistor arrays for the analysis of anti-cancer drug action on individual cells," Biosensors Bioelectron., vol. 40, no. 1, pp. 50-56, Feb. 2013, doi: 10.1016/j.bios.2012. 06.006.

[19] K. Hu, E. Kennedy, and J. K. Rosenstein, "High frequency dielectric spectroscopy array with code division multiplexing for biological imaging," in Proc. IEEE Biomed. Circuits Syst. Conf. (BioCAS), Montreal, QC, Canada, Oct. 2019, pp. 1-4, doi: 10.1109/BIOCAS.2019. 8919173.

[20] S. R. Mahmoodi, P. Xie, M. Allen, and M. Javanmard, "Multiwell plate impedance analysis of a nanowell array sensor for label-free detection of cytokines in mouse serum," IEEE Sensors Lett., vol. 4, no. 2, pp. 1-4, Feb. 2020, doi: 10.1109/LSENS.2020.2968214.

[21] S. Forouhi, R. Dehghani, and E. Ghafar-Zadeh, "Toward high throughput core-CBCM CMOS capacitive sensors for life science applications: A novel current-mode for high dynamic range circuitry," Sensors, vol. 18, no. 10, pp. 1-4, 2018, doi: 10.3390/s18103370.

[22] W. Tedjo and T. Chen, "An integrated biosensor system with a highdensity microelectrode array for real-time electrochemical imaging," IEEE Trans. Biomed. Circuits Syst., vol. 14, no. 1, pp. 20-35, Feb. 2020, doi: 10.1109/TBCAS.2019.2953579. 
[23] F. Pittino, P. Scarbolo, F. Widdershoven, and L. Selmi, "Derivation and numerical verification of a compact analytical model for the AC admittance response of nanoelectrodes, suitable for the analysis and optimization of impedance biosensors," IEEE Trans. Nanotechnol., vol. 14, no. 4, pp. 709-716, Jul. 2015, doi: 10.1109/TNANO.2015.2434106.

[24] J. C. Chen, B. W. McGaughy, D. Sylvester, and C. Hu, "An on-chip, attofarad interconnect charge-based capacitance measurement (CBCM) technique," in IEDM Tech. Dig., Dec. 1996, pp. 69-72, doi: 10.1109/IEDM. 1996.553124.

[25] F. Pittino and L. Selmi, "Use and comparative assessment of the CVFEM method for Poisson-Boltzmann and Poisson-Nernst-Planck three dimensional simulations of impedimetric nano-biosensors operated in the DC and AC small signal regimes," Comput. Methods Appl. Mech. Eng., vol. 278, pp. 902-923, Aug. 2014, doi: 10.1016/j.cma.2014.06.006.

[26] P. Scarbolo, F. Pittino, M. D. Longa, A. Cossettini, and L. Selmi (Apr. 2017). ENBIOS-1D Lab. [Online]. Available: https://nanohub.org/ resources/biolab, doi: 10.4231/D3Z02Z963.

[27] A. Hoxha, P. Scarbolo, A. Cossettini, F. Pittino, and L. Selmi. (Oct. 2016). ENBIOS-2D Lab. [Online]. Available: https://nanohub. org/resources/biolabisfet, doi: 10.4231/D3V11VM7D.

[28] A. P. Stogryn, H. T. Bull, K. Rubayi, and S. Iravanchy, "The microwave dielectric properties of sea and fresh water," GenCorp Aerojet, Azusa, CA, USA, Tech. Rep., 1995, p. 91702.

[29] E. Gongadze, U. van Rienen, and A. Iglic, "Generalized Stern models of the electric double layer considering the spatial variation of permittvity and finite size of ions in saturation regime," Cell. Mol. Biol. Lett., vol. 16, pp. 576-594, Aug. 2011, doi: 10.2478/s11658-011-0024-x.

[30] F. Pittino, P. Palestri, P. Scarbolo, D. Esseni, and L. Selmi, "Models for the use of commercial TCAD in the analysis of silicon-based integrated biosensors," Solid-State Electron., vol. 98, pp. 63-69, Aug. 2014. [Online]. Available: https://doi.org/10.1016/j.sse.2014.04.011

[31] Z. Zhu, X. Xu, L. Fang, D. Pan, and Q.-A. Huang, "Investigation of geometry-dependent sensing characteristics of microfluidic electrical impedance spectroscopy through modeling and simulation," Sens. Actuators B, Chem., vol. 235, pp. 515-524, Nov. 2016, doi: 10.1016/j. snb.2016.05.092.

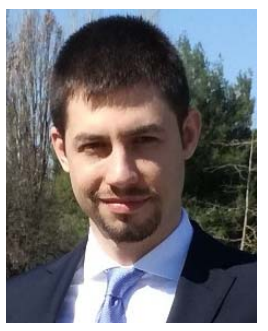

Andrea Cossettini (Member, IEEE) received the B.Sc., M.Sc. (cum laude), and Ph.D. degrees in electronic engineering from the University of Udine, Udine, Italy, in 2012, 2015, and 2019, respectively. In 2014, he was with Acreo Swedish ICT AB, Kista, Sweden, designing waveguideto-chip transitions at sub-mm waves. In 2015 he was with Infineon Technologies, Villach, Austria, working on signal integrity for high-speed serial interfaces. During the Ph.D., he worked on nanoelectrode array biosensors for highfrequency impedance spectroscopy and imaging. He is currently a Postdoctoral Researcher with ETH Zürich, Zürich, Switzerland, working on the design of circuits and systems for biomedical applications.

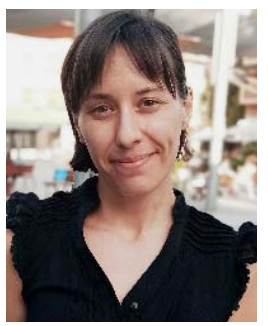

Cecilia Laborde is a Data Scientist in the private sector. Her work focuses on developing physical and machine learning models for a wide variety of topics extending from equipment performance in the mining industry to shift demand forecasting of healthcare institutions.

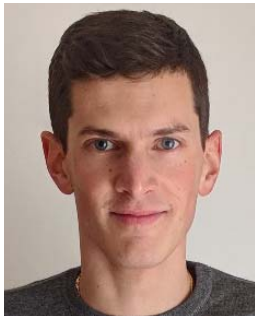

Denis Brandalise (Student Member, IEEE) received the M.Sc. (cum laude) degree in electronic engineering from the University of Udine, Udine, Italy, in 2018. He is currently pursuing the Ph.D. degree in characterization of massively parallel impedance spectroscopy electronic nanobiosensors. In 2018, while focusing on modeling the effects of different receptor-surface binding modes on the electrochemical properties of peptide-nucleic-acid-based sensing platforms, he spent four months at the Walter Schottky Institute, Garching bei München, Germany.

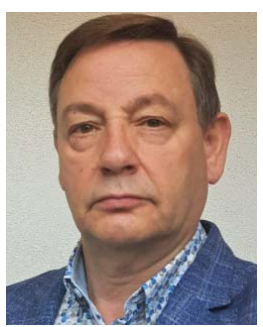

Frans Widdershoven received the M.Sc. (cum laude) degree from the Eindhoven University of Technology, Eindhoven, The Netherlands, in 1984, and the Ph.D. degree from the University of Twente, Enschede, The Netherlands, in 1991. From 1984 to 2006, he was with the Philips Research. Since 2006, he has been with NXP Semiconductors, where he has also been a Fellow since 2010. Since December 2019, he has also been a part-time Full Professor with the Bioelectronics Group, Faculty EEMCS, Delft University of Technology, The Netherlands. He has coauthored 73 journal, conference, and book contributions, and holds 45 granted U.S. patents. His research interests include silicon process technology and device physics, low-power IC design, signal processing, machine learning, and embedded pixelated capacitive sensor applications.

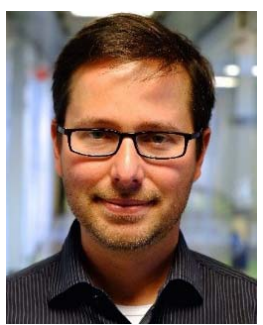

Serge G. Lemay received the B.A.Sc. degree in electrical engineering with minor in physics from the University of Waterloo, Canada, in 1993, and the Ph.D. degree in physics from Cornell University, USA, in 1999. He was a Faculty Member with the Delft University of Technology, The Netherlands, from 2001 to 2009. In 2009, he relocated to the University of Twente, The Netherlands, where he heads the Bioelectronics Group. His research has spanned solid-state physics, molecular electronics, and biophysics. His research interests include the fundamentals of electron transfer in liquid and exploring digital transduction mechanisms for biosensing.

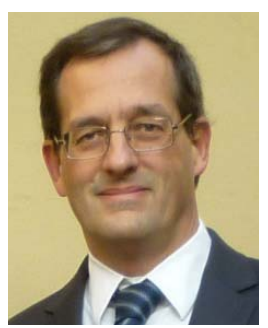

Luca Selmi (Fellow, IEEE) received the Ph.D. degree in electronic engineering from the University of Bologna, Bologna, Italy, in 1992. Since 2000, he has been a Professor of electronics with the University of Udine, Udine, Italy, and then recently moved to the University of Modena and Reggio Emilia. His research interests include simulation, modeling, and characterization of nanoscale CMOS transistors and NVM, with emphasis on hot-carrier effects, quasiballistic transport, Monte Carlo simulation techniques, and with a recent twist toward nanoelectronic (bio)sensors, simulation and characterization of ISFET, and impedance spectroscopy sensors. He was a TPC member of various conferences, including IEEE IEDM and IEEE Symposium on VLSI Technology, and the Editor of IEEE ELECTRON DEVICE LETTERS (EDL). 